\title{
A NOTE ON THE STRUCTURE OF NORMAL HAMILTONIAN MATRICES
}

\section{CHRistos CHORIANOPOUlOS}

Abstract. The structures of the blocks of a normal Hamiltonian matrix are studied. In this note it is obtained that all four blocks of a normal Hamiltonian matrix $H=\left[\begin{array}{cc}A & B \\ C & -A^{*}\end{array}\right]$ can be expressed as linear combinations of four other matrices.

Mathematics subject classification (2020): 15A21, 15B99, 47A15.

Keywords and phrases: Hamiltonian matrix, normal matrix.

\section{REFERENCES}

[1] Silvia Gigola, Leila LebTahi And Nestor Thome, Inverse eigenvalue problem for normal J-Hamiltonian matrices, Applied Mathematics Letters 48 (2015), 36-40.

[2] Peter Lancaster, Stability of linear gyroscopic systems: A review, Linear Algebra and its Applications 439 (3) (2013), 686-706.

[3] Wen Wei Lin, Volker Mehrmann and Hongguo Xu, Canonical Forms for Hamiltonian and Sympletic Matrices and Pencils, Linear Algebra and its Applications 302-303 (1999), 469-533.

[4] Chris Paige ANd Charles VAN LOAN, A Schur decomposition for Hamiltonian matrices, Linear Algebra and its Applications 41 (1981), 11-32.

[5] JiA ZHAO AND JIEMING ZHANG, The solvability conditions for the inverse eigenvalue problem of normal skew J-Hamiltonian matrices, Journal of Inequalities and Applications 2018:74, 2018. 